Bundesgesundheitsbl 2014 -57:747-761

DOI 10.1007/s00103-014-1973-9

(c) Springer-Verlag Berlin Heidelberg 2014
M. Lange - H. G. Butschalowsky · F. Jentsch - R. Kuhnert • A. Schaffrath Rosario • M. Schlaud · P. Kamtsiuris · KiGGS Study Group

Abteilung für Epidemiologie und Gesundheitsberichterstattung, Robert Koch-Institut, Berlin

\title{
Die erste KiGGS-Folgebefragung (KiGGS Welle 1)
}

\section{Studiendurchführung, Stichprobendesign und Response}

\section{Hintergrund und Fragestellung}

Die „Studie zur Gesundheit von Kindern und Jugendlichen in Deutschland“ (KiGGS) ist Bestandteil des Gesundheitsmonitorings, welches das Robert KochInstitut (RKI) im Auftrag des Bundesministeriums für Gesundheit durchführt $[1,2]$. Ein Ziel von KiGGS ist es, regelmäßig bevölkerungsbezogene Daten zur gesundheitlichen Lage der in Deutschland lebenden Kinder und Jugendlichen unter 18 Jahren zu erheben [3]. Das Erhebungsspektrum umfasst vielfältige Informationen $\mathrm{zu}$ Gesundheitsstatus und -verhalten, Lebensbedingungen sowie Inanspruchnahme von Leistungen des Gesundheitssystems. Die Daten werden mittels Befragungen sowie - in bestimmten Zeitabständen - mittels körperlicher Untersuchungen, Tests und Laboranalysen von Blut- und Urinproben gewonnen. Sie erlauben es, innerhalb der Erhebungswellen verschiedene Personengruppen hinsichtlich der erhobenen Merkmale zu vergleichen (Querschnittanalysen) und durch den Vergleich der KiGGS-Daten verschiedener Erhebungswellen Aussagen über die Entwicklung der gesundheitlichen Lage im Zeitverlauf zu treffen (Trendanalysen). Ein weiteres Ziel von KiGGS ist es, durch die wiederholte Einbeziehung der Teilnehmenden der Basiserhebung (KiGGS-Kohorte) längsschnittliche Daten zu sammeln, die Erkenntnisse über die Ursachen und Bedingungen gesundheitlicher Veränderungen im Le- bensverlauf - insbesondere bis ins junge Erwachsenenalter - ermöglichen.

Die KiGGS-Daten fließen in die Gesundheitsberichterstattung des Bundes am RKI ein. Sie werden für die epidemiologische und die Public-Health-Forschung genutzt und auf Antrag Interessenten aus der wissenschaftlichen Community durch das Forschungsdatenzentrum am RKI zur Verfügung gestellt. Die Studienergebnisse sind eine wichtige Grundlage für die Gesundheitspolitik und die evidenzbasierte Konzeption von Präventions- und Interventionsmaßnahmen.

Die KiGGS-Basiserhebung führte das RKI von 2003 bis 2006 in 167 Untersuchungsorten (Sample Points) als kombinierten Untersuchungs- und Befragungssurvey durch [4-6]. Die erste Folgebefragung - genannt KiGGS Welle 1 - erfolgte von 2009 bis 2012. Der vorliegende Beitrag ergänzt die themenspezifischen Beiträge zu KiGGS Welle 1 im vorliegenden Heft und liefert methodische Informationen, die für die Interpretation der Studienergebnisse grundlegend sind. Dargestellt werden im Abschnitt Methoden zum einen zentrale Aspekte der Stichprobenziehung, Teilnehmendengewinnung und der Datenerhebung, zum anderen die eingesetzten methodischen Instrumente zur Beurteilung möglicher Stichprobenverzerrungen sowie das Berechnungsverfahren für Gewichtungsfaktoren. Anschließend werden im Abschnitt Ergebnisse die Gründe für qualitätsneutrale Ausfälle und für die Nichtteilnahme (Nonresponse) erläutert sowie Kenn- zahlen zur Beteiligung (Response), Wiederteilnahmebereitschaft und Aspekte der Repräsentativität beleuchtet. In der abschließenden Diskussion werden verschiedene Aspekte des Studiendesigns im Hinblick auf ihre möglichen Auswirkungen auf die Ergebnisse diskutiert. Die gesamte Darstellung bezieht sich ausschließlich auf den für Prävalenzschätzungen im Kindes- und Jugendalter relevanten Altersbereich von 0 bis 17 Jahren (• Abb. 1). Nicht berücksichtigt werden hierbei die Teilnehmenden der Kohorte im Alter von 6 Jahren, da dieser Altersjahrgang für den Querschnitt durch die neue Stichprobe abgedeckt ist.

\section{Methoden}

\section{Stichprobendesign}

Zielpopulation für Prävalenzschätzungen bei Kindern und Jugendlichen aus KiGGS Welle 1 waren die im Erhebungszeitraum 2009 bis 2012 in Deutschland lebenden und in den Einwohnermelderegistern mit Hauptwohnsitz gemeldeten Kinder und Jugendlichen, die zum jeweiligen Befragungszeitpunkt unter 18 Jahre alt waren. Hierfür wurde ein komplexes, mehrstufiges und geschichtetes Stichprobendesign gewählt, das in Kooperation mit dem Zentrum für Umfragen, Methoden und Analysen (ZUMA) Mannheim (seit 2008

Die KiGGS Study Group: Die Abteilung Epidemiologie und Gesundheitsmonitoring im Robert Koch-Institut. 


\section{Leitthema}

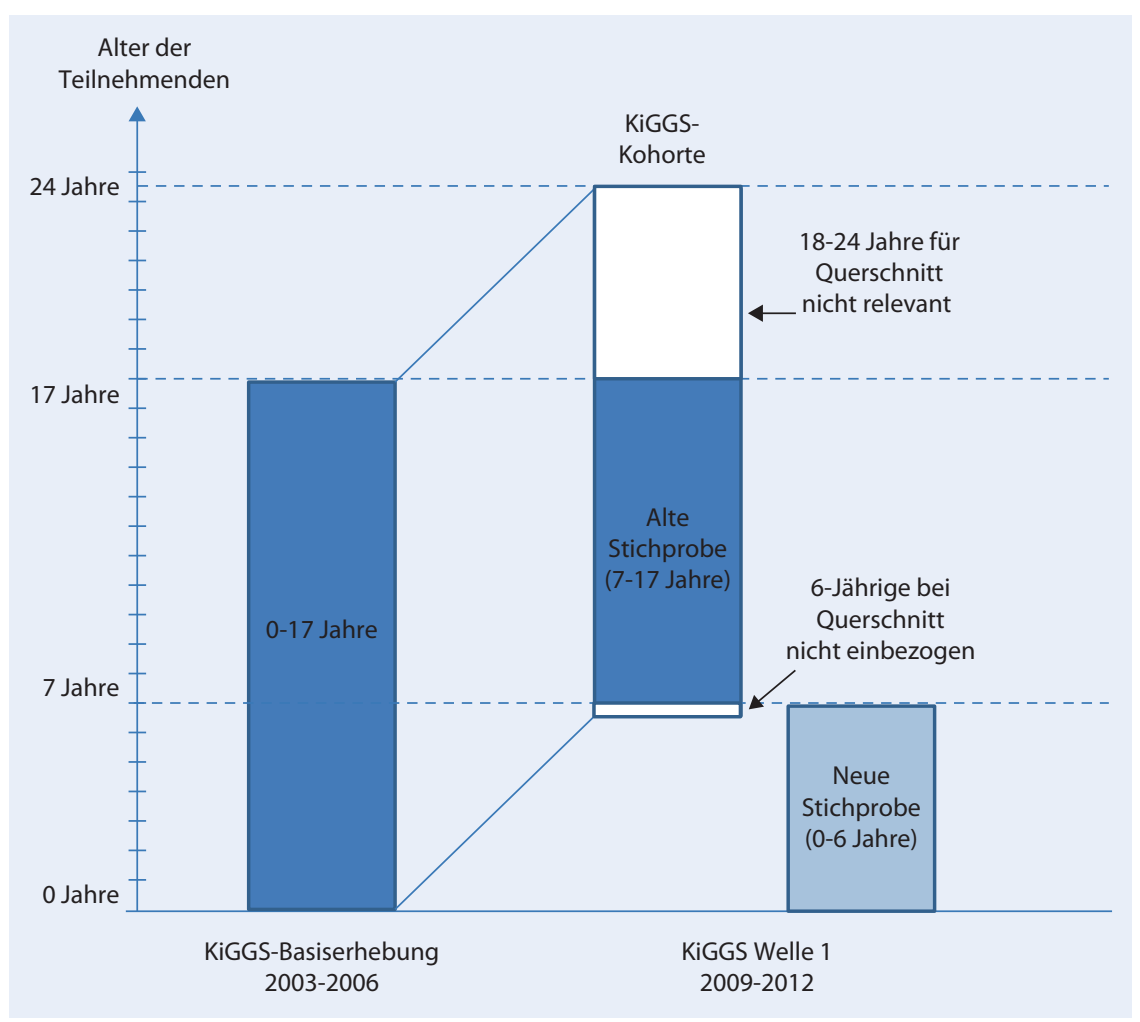

Abb. $1 \Delta$ Stichprobendesign

GESIS - Leibniz-Institut für Sozialwissenschaften) bereits für die KiGGS-Basiserhebung entwickelt worden war [5]:

\section{Erste Stufe: Auswahl der Sample Points}

Für die KiGGS-Basiserhebung wurde zunächst aus allen Städten und Gemeinden in Deutschland eine nach Bundesländern und Gemeindetypen (10-stufige BIKKlassifikation [7]) proportional geschichtete Zufallsstichprobe von 167 Untersuchungsorten („Sample Points“) gezogen, wobei für die „neuen“ Bundesländer ein disproportionales Oversampling etwa um den Faktor 2 vorgenommen wurde, um ausreichende Fallzahlen für Ost/WestVergleiche zu erlangen. Diese 167 Studienorte wurden auch für KiGGS Welle 1 beibehalten.

\section{Zweite Stufe: Auswahl der Zielpersonen}

Für die als Querschnittstudie konzipierte KiGGS-Basiserhebung wurde im zweiten Schritt für jeden Altersjahrgang der 0bis einschließlich 17-Jährigen eine gleiche Anzahl von Personen als uneingeschränkte Zufallsauswahl aus den Melderegistern aller 167 Sample Points gezogen und mit ihren Eltern zur Studienteilnahme eingeladen [5]. Um die erfahrungsgemäß hohe Quote qualitätsneutraler Ausfälle und die niedrigere Teilnahmebereitschaft bei Personen mit Migrationshintergrund zu kompensieren, erfolgte ein Oversampling von Personen mit nichtdeutscher Staatsangehörigkeit etwa um den Faktor 1,5.

Für KiGGS Welle 1 wurde dieses Stichprobenverfahren zwecks Gewinnung einer neuen Querschnittstichprobe von 0 - bis einschließlich 6-Jährigen in identischer Weise durchgeführt. Darüber hinaus wurden alle ehemaligen Teilnehmenden der KiGGS-Basiserhebung, die inzwischen von 6 bis einschließlich 24 Jahre alt waren und als geschlossene Kohorte weitergeführt werden, zur Befragung eingeladen, sofern sie nicht einer erneuten Einladung widersprochen hatten, ins Ausland verzogen oder verstorben waren. Wiedereingeladene, die innerhalb Deutschlands aus dem Sample Point ihrer Erstteilnahme verzogen waren, wurden nicht von der Befragung ausgeschlossen. Das Stichprobendesign für KiGGS Welle 1 wurde ebenfalls mit dem Leibniz-Institut für Sozialwissenschaften (GESIS) abgestimmt.
Teilnehmendengewinnung und Datenerhebung

Vor Beginn der Studie lagen positive Voten der Ethikkommission der Charité Universitätsmedizin Berlin und des Bundesbeauftragten für den Datenschutz vor.

\section{Zeitliche Abfolge der Datenerhebungen in den Sample Points}

Die Datenerhebungen zu KiGGS Welle 1 erstreckten sich von Juni 2009 bis Juni 2012. Dabei wurden die 167 Untersuchungsorte - bis auf ferienbedingte Ausnahmen - in derselben Reihenfolge einbezogen wie schon in der KiGGS-Basiserhebung, um möglichst gleiche Abstände zwischen den beiden Erhebungszeitpunkten zu gewährleisten und erneut saisonale und regionale Einflüsse auf die Datenerhebung auszuschließen [4]. Für jeden Ort wurde ein dreiwöchiger Erhebungszeitraum festgelegt, in dem der Großteil der Telefoninterviews absolviert werden sollte. Das Alter der Teilnehmenden wurde mit Bezug auf das tatsächliche Interviewdatum berechnet (falls sowohl ein Kinder-/Jugendlicheninterview und ein Elterninterview vorlagen, wurde das Datum des Kinder-/Jugendlicheninterviews zugrunde gelegt).

\section{Einwilligungsverfahren}

Für die Befragung von Kindern und Jugendlichen sowie für Erhebungen, die auf Einwohnermeldeamtsstichproben basieren, gelten besondere datenschutzrechtliche Voraussetzungen. So müssen die potenziellen Teilnehmenden - im Gegensatz zu reinen Telefonnummernstichproben - zuvor schriftlich über die Studie informiert werden. Zudem ist eine Befragung Minderjähriger nur zulässig, wenn die Sorgeberechtigten hierzu ihr schriftliches Einverständnis gegeben haben.

\section{Schriftliche Einladung und Erinnerung}

In der Regel 6 Wochen vor Beginn des Befragungszeitraums im jeweiligen Sample Point wurden die Eltern durch die KiGGS-Geschäftsstelle schriftlich über die Studie informiert und zur Teilnahme eingeladen (s. - Abb. 2). Die Befragung der Eltern und - ab 11 Jahren - der 
Kinder und Jugendlichen erfolgte nach Rücksendung der von den Sorgeberechtigten (zumeist den Eltern) unterzeichneten Einwilligungserklärung. War nach 10 Tagen keine $\mathrm{Zu}$ - oder Absage eingegangen, wurde ein Erinnerungsschreiben versendet.

\section{Telefonische Erinnerung}

Sofern nach weiteren 10 Tagen keine Antwort vorlag, wurden die Eingeladenen telefonisch kontaktiert. Dafür mussten für die neue Querschnittstichprobe die Rufnummern aus öffentlich zugänglichen Verzeichnissen recherchiert werden (s. Abschnitt Gründe für die Nichtteilnahme). Für die Teilnehmenden der KiGGS-Kohorte lagen überwiegend noch gültige Rufnummern aus der Basiserhebung vor. Die Anrufe erfolgten durch geschulte Interviewerinnen und Interviewer des RKI-Telefonlabors, die auch die telefonischen Befragungen durchführten. Um eine Zielperson zu erreichen, wurden maximal 15 Kontaktversuche an verschiedenen Tagen und zu unterschiedlichen Zeiten unternommen. Teilnahmebereite Eltern wurden gebeten, zunächst die schriftliche Einwilligungserklärung zurückzusenden und danach das Interview durchzuführen. Auf ausdrücklichen Wunsch mancher Eltern konnte auch sofort ein sog. „Impuls-Interview“ absolviert werden. Sofern die Einwilligungserklärung nicht nachgereicht wurde, wurden die Erhebungsdaten der ImpulsInterviews gelöscht. Mit Kindern und Jugendlichen wurden keine Impuls-Interviews durchgeführt.

\section{Datenerhebung}

Bei KiGGS Welle 1 wurden standardisierte Telefoninterviews mit den Eltern der 0bis 17-Jährigen und - im Alter von 11 bis 17 Jahren - mit den Kindern und Jugendlichen selbst geführt. Dabei wurden nahezu alle Befragungsthemen aus den Fragebögen der KiGGS-Basiserhebung nach Anpassung an die Erfordernisse der Telefonbefragung übernommen. In wenigen Fällen wurden die ursprünglichen Instrumente durch geeignetere ersetzt, teilweise wurden zusätzliche Instrumente aufgenommen [3] (für eine detaillierte Themenübersicht s. [8]).

Bundesgesundheitsbl 2014 · 57:747-761 DOI 10.1007/s00103-014-1973-9

(c) Springer-Verlag Berlin Heidelberg 2014

M. Lange · H. G. Butschalowsky · F. Jentsch • R. Kuhnert • A. Schaffrath Rosario · M. Schlaud • P. Kamtsiuris · KiGGS Study Group

Die erste KiGGS-Folgebefragung (KiGGS Welle 1). Studiendurchführung, Stichprobendesign und Response

\section{Zusammenfassung}

Die „Studie zur Gesundheit von Kindern und Jugendlichen in Deutschland" (KiGGS) ist Bestandteil des Gesundheitsmonitorings des Robert Koch-Instituts (RKI). Nach der KiGGSBasiserhebung (2003-2006), die Untersuchungen und Befragungen von Kindern und Jugendlichen im Alter von 0 bis 17 Jahren umfasste, wurde KiGGS Welle 1 (2009-12) als Telefonbefragung durchgeführt. Neben der Bereitstellung längsschnittlicher Daten besteht eine zweite zentrale Zielsetzung von KiGGS darin, für den Altersbereich von 0 bis 17 Jahren wiederholt bundesweite, bevölkerungsbezogene Querschnittdaten zur gesundheitlichen Lage bereitzustellen. Das Stichprobendesign von KiGGS Welle 1 sah deshalb neben der Wiedereinladung von Teilnehmenden der KiGGS-Basiserhebung (KiGGS-Kohorte) die Ziehung einer neuen Einwohnermeldeamtsstichprobe 0- bis 6-jähriger Kinder aus den 167 Studienorten vor, da dieser Altersbereich durch die älter gewordenen Wiedereingeladenen nicht mehr besetzt war. Der vorliegende Beitrag konzentriert sich auf den für Prävalenzschätzungen im Kindes- und Jugendalter relevanten Altersbereich von 0 bis 17 Jahren. Insgesamt nahmen 12.368 Kinder und Jugendliche unter 18 Jahren teil, darunter 4455 Ersteingeladene und 7913 Wiedereingeladene (Response 38,8 und 72,9\%). Der Vergleich der Nettostichprobe mit der Bevölkerung Deutschlands ( 0 bis 17 Jahre) bezüglich verschiedener Merkmale und eine Analyse des Zusammenhangs zwischen der Wiederteilnahmerate und in der KiGGS-Basiserhebung erhobenen Merkmalen bei den 7- bis 17-Jährigen weisen auf eine verzerrungsarme Stichprobe hin. Zur Anpassung der Stichprobe hinsichtlich einzelner Merkmale an die Bevölkerungsstruktur sowie zum partiellen Ausgleich der Nonresponse wurden für Querschnitt- und Trendanalysen jeweils Gewichtungsfaktoren berechnet.

\section{Schlüsselwörter}

Gesundheitssurvey · Kinder und Jugendliche . Stichprobe $\cdot$ Response $\cdot$ Gewichtung

\section{The first KiGGS follow-up (KiGGS Wave 1). Study conduct, sample design, and response}

\section{Abstract}

The "German Health Interview and Examination Survey for Children and Adolescents" (KiGGS) is part of the health monitoring system of the Robert Koch Institute (RKI). Following the KiGGS baseline study (2003-06), which comprised interviews and physical examinations of 0 - to 17-year-old participants, KiGGS Wave 1 (2009-2012) was carried out as a telephone-based survey. In addition to providing longitudinal data, a second essential aim of KiGGS is to regularly provide population-based cross-sectional data on the health situation of children and adolescents aged 0-17 years living in Germany. Therefore, the study population of KiGGS Wave 1 consists of re-invited participants from the baseline study (KiGGS cohort), supplemented by newly invited children aged 0-6 years. The newly invited participants were randomly chosen from local population registries in the 167 baseline sample points. This method was chosen to supplement the sample with

\begin{abstract}
younger age groups. This article focuses on the age groups from 0 to 17 years, which are relevant for prevalence estimations among children and adolescents. In total 12,368 children and adolescents took part; among them 4,455 newly invited and 7,913 re-invited participants (response 38.8 and $72.9 \%$, respectively). A comparison of the net sample with the resident German population ( $0-17$ years) regarding particular population characteristics and an analysis of the relationship between the re-participation rate and certain characteristics collected in the baseline study (7-17 years) suggest a mostly unbiased sample. To account for certain aspects of the population and nonresponse, cross-sectional and trend analyses were partially corrected by weighting factors.
\end{abstract}

\section{Keywords}

Health survey - Children and adolescents . Sample $\cdot$ Response $\cdot$ Weighting 


\section{Leitthema}

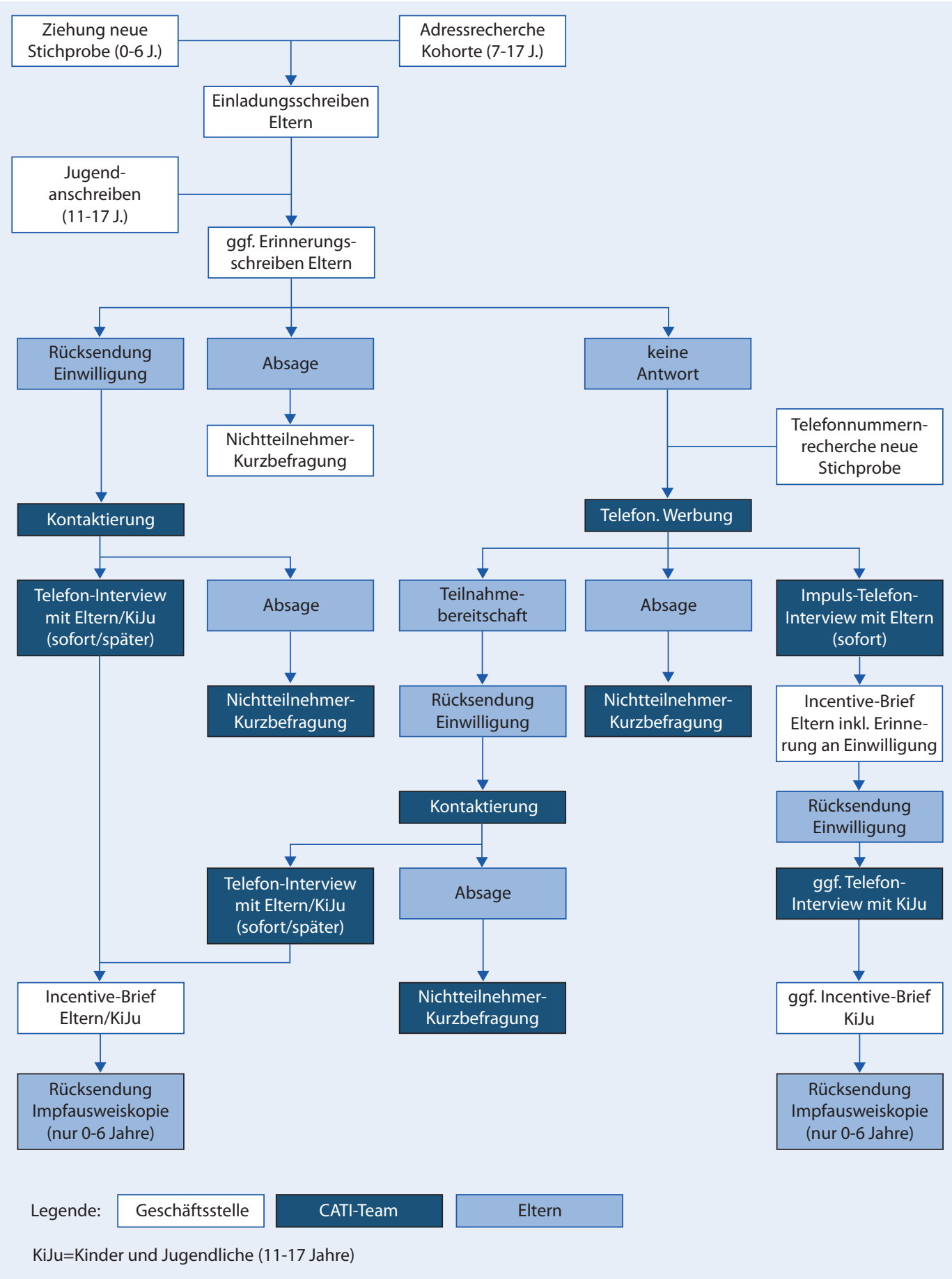

Abb. $2 \triangleleft$ Ablauf der Teilnehmendengewinnung und Feldarbeit

Die Befragungen erfolgten in der Regel als Computer Assisted Telephone Interview (CATI) durch 42 geschulte Interviewerinnen und Interviewer im RKI-Telefonlabor [9] (zum Einsatz von Papierfragebögen s. unten). Die Durchführung der standardisierten Interviews wurde durch erfahrene Supervisoren begleitet und qualitätsgesichert. Die Interviews mit Kindern und Jugendlichen dauerten durchschnittlich $30 \mathrm{~min}$, die Interviews mit den Sorgeberechtigten (zumeist den Eltern) etwa $40 \mathrm{~min}$. Die Anrufe erfolgten in der Regel in den von den Befragten gewünschten Zeitfenstern bzw. zu vereinbarten Terminen. Es stand den Eltern frei, welcher Elternteil die Fragen beantwortet.

\section{Maßnahmen zur Verbesserung der Teilnahmebereitschaft}

Um eine hohe Teilnahmequote sicherzustellen, wurden die folgenden Maßnahmen umgesetzt:

- Die Namen und Anschriften zur Gewinnung der neuen Querschnittstichprobe wurden möglichst kurz vor dem Einladungstermin vom je- 
weiligen Meldeamt zufällig gezogen. Die Anschriften von Teilnehmenden der KiGGS-Kohorte wurden kurz vor Versendung der Einladungsschreiben über die Meldeämter auf Gültigkeit geprüft.

- Ersteingeladene Familien erhielten mit der Einladung zusätzlich eine Broschüre mit Ergebnissen der vorhergehenden KiGGS-Basiserhebung [10].

- Ergänzende Informationen zur Studie und zur durchführenden Institution waren auf der Studien-Website (www.kiggs-studie.de) und auf der RKI-Website verfügbar. Überdies konnten sich die Eingeladenen telefonisch (gebührenfrei) oder per E-Mail mit Fragen an die KiGGS-Geschäftsstelle wenden.

- Um auch Kinder und Jugendliche mit Migrationshintergrund in ausreichendem Maße zu beteiligen, wurden die Namen der Kinder und Jugendlichen zur Gewinnung der neuen Querschnittstichprobe mittels eines onomastischen Zuordnungsverfahrens auf mutmaßliche nichtdeutsche Herkunft geprüft. Im positiven Falle oder einer nichtdeutschen Staatsangehörigkeit erhielten die Eltern neben der deutschen Einladung auch ein gleichlautendes Schreiben auf Arabisch, Englisch, Russisch, Serbokroatisch, Türkisch oder Vietnamesisch. Auch die Eltern von wiedereingeladenen Kohortenteilnehmenden erhielten Anschreiben in ihrer Herkunftssprache. Da die Telefoninterviews ausschließlich in deutscher Sprache realisiert werden konnten, hatten Eltern mit nicht ausreichenden Deutschkenntnissen die Möglichkeit, schriftliche Fragebögen auf Deutsch oder in einer der oben genannten Sprachen zu verwenden.

- Der Einladungsprozess wurde flankiert durch eine studienortbezogene Öffentlichkeitsarbeit über lokale Medien, sodass insbesondere ersteingeladene Familien im besten Falle schon etwas über KiGGS Welle 1 gehört oder gelesen hatten, wenn sie das Einladungsschreiben erhielten [8]. Zusätzlich wurden die in den Studien- orten niedergelassenen Kinderärzte vorab über die Studie informiert.

- Nach Abschluss der Telefoninterviews erhielten alle befragten Eltern sowie Kinder und Jugendlichen als Aufwandsentschädigung einen Einkaufsgutschein im Wert von 10 Euro.

- Bei einer Unterstichprobe der Teilnehmenden wurde nach Abschluss der Interviews eine Online-Befragung zur Zufriedenheit durchgeführt [11].

Bestimmung der Teilnahmebereitschaft und Berechnung von Gewichtungsfaktoren

Für die Einschätzung möglicher Verzerrungen der Prävalenzschätzer durch unterschiedliche Teilnahmebereitschaften von Untergruppen (Self-Selection Bias) bei den 0- bis 17-jährigen Teilnehmenden wurde zunächst die Teilnahmequote nach Untergruppen bestimmt und die Verteilung von Basischarakteristika zwischen Studienpopulation und Grundgesamtheit (amtliche Statistik) geprüft. Als spezielle gesundheits- bzw. versorgungsbezogene Indikatoren wurden die Krankenversicherung und der Anteil von Personen mit amtlich anerkannter Schwerbehinderung herangezogen, weil beide Merkmale aus bevölkerungsbezogenen Datenquellen bekannt sind.

Für die wiedereingeladenen Kinder und Jugendlichen der Kohorte standen darüber hinaus die Erhebungsdaten aus der Basiserhebung zur Verfügung. Diese wurden herangezogen, um für die Stichprobe der 7 bis 17-Jährigen

1. sowohl eine deskriptive univariate als auch eine modellbasierte multivariate Analyse des Zusammenhangs zwischen der Wiederteilnahmewahrscheinlichkeit und den erhobenen Merkmalen in der KiGGS-Basiserhebung durchzuführen sowie

2. die Unterschiede zwischen Respondern und Nonrespondern hinsichtlich vorhandener Zielindikatoren zu analysieren und dann variablenbezogen den deterministischen Nonresponse-Bias zu berechnen und zu bewerten.
Der Nonresponse-Bias ist hierbei eine Funktion der Nonresponse-Quote [1Response $\left(t_{R}\right)$ ] und der Differenz der zu erhebenden Surveyvariablen (Zielvariablen) zwischen Respondern $\left(\bar{Y}_{R}\right)$ und Nonrespondern $\bar{Y}_{N R}[12]$.

$$
B_{N R}=\left(1-t_{R}\right)\left(\bar{Y}_{R}-\bar{Y}_{N R}\right)
$$

\section{Berechnung der Gewichtungsfaktoren}

Für Querschnittanalysen der beiden Stichproben von KiGGS Welle 1 wurden jeweils Gewichtungsfaktoren berechnet, die sich aus einem Design- und einem Anpassungsgewicht zusammensetzen.

\section{Designgewicht für die neuen Querschnittteilneh- menden ( 0 bis 6 Jahre)}

Die Designgewichte für die neue Querschnittstichprobe entsprechen den inversen Auswahlwahrscheinlichkeiten der Teilnehmenden. Die Auswahlwahrscheinlichkeiten sind gegeben durch das Produkt der Auswahlwahrscheinlichkeit des jeweiligen Sample Points und der Auswahlwahrscheinlichkeit der teilnehmenden Personen innerhalb des Sample Points. Hierbei erfolgte - getrennt für die 3 Regionen West, Ost und Berlin - die Bestimmung der Auswahlwahrscheinlichkeit des Sample Points aus dem Bevölkerungsumfang der 0- bis 17-Jährigen im Sample Point dividiert durch den Bevölkerungsumfang der jeweiligen Region (Bevölkerungsstand 31.12.2000). Die Auswahlwahrscheinlichkeit der Teilnehmenden innerhalb eines Sample Points wiederum ergab sich aus der Zahl der teilnehmenden Personen pro Altersjahrgang dividiert durch die Gesamtzahl der in dem entsprechenden Altersjahrgang lebenden Kinder in der Gemeinde (bezogen auf den jeweiligen Erhebungszeitraum). Die Auswahlwahrscheinlichkeiten wurden also auf Basis der Nettostichprobe berechnet, und die Designgewichte gleichen damit neben den durch Stratifizierung und Clusterung bedingten ungleich großen Auswahlwahrscheinlichkeiten auch die nach Alter, Region und Gemeindegröße variierende Nonresponse aus. 
Tab. 1 Übersicht mit Kennzahlen zur Teilnehmenden-Gewinnung bei KiGGS Welle 1

\begin{tabular}{|c|c|c|c|}
\hline & $\begin{array}{l}\text { Alte Stichprobe (ehe- } \\
\text { malige Teilnehmende } \\
\text { der KiGGS-Basiserhe- } \\
\text { bung) }\end{array}$ & $\begin{array}{l}\text { Neue Einwohner- } \\
\text { meldeamtsstichpro- } \\
\text { be ( } 0 \text { bis } 6 \text { Jahre) }\end{array}$ & Gesamt \\
\hline $\begin{array}{l}\text { Teilnehmende der KiGGS- } \\
\text { Basiserhebung }\end{array}$ & 17.641 & - & - \\
\hline $\begin{array}{l}\text { Teilnehmende der KiGGS- } \\
\text { Basiserhebung, die im KiGGS } \\
\text { Welle 1-Erhebungszeitraum } 7 \\
\text { bis } 17 \text { Jahre alt waren }\end{array}$ & 10.924 & - & - \\
\hline QNA vor Studienbeginn 2009 & 61 & - & - \\
\hline Unbereinigte Bruttostichprobe & 10.863 & 11.834 & 22.697 \\
\hline QNA im Verlauf der Feldarbeit & 8 & 349 & 357 \\
\hline Bereinigte Bruttostichprobe & 10.855 & 11.485 & 22.340 \\
\hline Nichtteilnehmende & 2942 & 7030 & 9972 \\
\hline Teilnehmende & 7913 & 4455 & 12.368 \\
\hline Response (\%) & 72,9 & 38,8 & - \\
\hline \multicolumn{4}{|l|}{ Teilnehmende pro Sample Point: } \\
\hline Durchschnitt & 47,4 & 26,7 & 74,1 \\
\hline 0,25 . Quartil & 42 & 22 & 64 \\
\hline 0,75 . Quartil & 53 & 32 & 84 \\
\hline
\end{tabular}

Wiederteilnahmegewicht für die Kohorteteilnehmenden (7 bis 17 Jahre)

Die Wiederteilnahmewahrscheinlichkeit in KiGGS Welle 1 wurde über eine mit dem KiGGS-Basis-Gewicht gewichtete logistische Regression mit der Wiederteilnahme als Zielvariable und verschiedenen Variablen der Basiserhebung (s. Ergebnisteil und - Tab.4) als Einflussgrößen geschätzt. Der Kehrwert der Wiederteilnahmewahrscheinlichkeit multipliziert mit dem KiGGS-Basis-Gewicht entspricht dem Wiederteilnahmegewicht der Kohortenteilnehmenden. Um extreme Gewichte zu vermeiden, wurde das Wiederteilnahmegewicht auf das 0,5\%-und 99,5\%-Quantil gestutzt.

\section{Anpassungsgewicht für die Gesamtstichprobe (0 bis 17 Jahre)}

Nachdem die Design- und Wiederteilnahmegewichte beider Stichproben zunächst auf den Gesamtstichprobenumfang normiert wurden, erfolgte eine Anpassung an die amtlichen Bevölkerungszahlen (Stand 31.12.2010) und die Bildungsverteilung des Haushaltsvorstands im Mikrozensus (2009, eingeschränkt auf Kinder unter 18 Jahren). Hierbei wurden war in 4 Ebenen unterteilt. In der ersten Ebene wurden Alter (nach Jahrgängen) und Geschlecht angepasst. In der zweiten Ebene ist die Bevölkerungsverteilung nach Bundesland, Geschlecht und Altersgruppe ( 0 bis 2, 3 bis 6,7 bis 10,11 bis 13 , 14 bis 17 Jahre) enthalten. Die dritte Ebene passt die Staatsangehörigkeit (deutsch $\mathrm{ja} /$ nein) nach West/Ost/Berlin gekreuzt mit der Altersgruppe ( 0 bis 6, 7 bis 17 Jahre) an. In der letzten Ebene wurde die Bildung (CASMIN-Klassifikation [13]) nach West/Ost/Berlin gekreuzt mit der Altersgruppe ( 0 bis 6,7 bis 13, 14 bis 17 Jahre) an den Mikrozensus 2009 angepasst. Die 4 Ebenen wurden nacheinander so lange wiederholt, bis die Änderung der Gewichte nur geringfügig war. Jeweils nach der vierten Ebene wurden die Gewichte innerhalb der Straten (West + Berlin und Ost) auf das 0,5\%- und 99,5\%-Quantil gestutzt.
Erstellung eines neuen Gewichts für die KiGGS-Basiserhebung

In der Gewichtungsvariablen für die KiGGS-Basiserhebung war der Bildungsstand der Eltern bislang nicht berücksichtigt worden. Es wurde daher für die Basiserhebung eine neue Gewichtungsvariable berechnet, welche das ursprüngliche Designgewicht verwendet und darauf eine Anpassungsgewichtung analog zu KiGGS Welle 1 aufsetzt (bezogen auf den Bevölkerungsstand 31.12.2004 und die Bildungsverteilung des Haushaltsvorstands aus dem Mikrozensus 2005). Für die Durchführung von Trendanalysen wurde zudem ein Gewicht berechnet, das die KiGGS-Basiserhebung bezüglich Bundesland, Altersjahrgang, Geschlecht und Staatsangehörigkeit auf den Bevölkerungsstand zum 31.12.2010 standardisiert.

Die statistischen Kennzahlen für die Gewichtungsvariablen können in einer Online-Tabelle unter http://www.kiggsstudie.de/kiggs-w1-supplements nachgelesen werden.

\section{Ergebnisse}

\section{Eingeladene Personen}

Zur Gewinnung der neuen Querschnittteilnehmenden wurden insgesamt 11.834 Einladungen an Eltern von Kindern unter 7 Jahren versendet, darunter 1194 Einladungen mit zusätzlicher Übersetzung.

Von den 17.641 Teilnehmenden der KiGGS-Basiserhebung waren zu Beginn von KiGGS Welle 110.924 im relevanten Alter von 7 bis 17 Jahren (• Tab. 1). Von diesen waren laut Vorrecherchen 60 ins Ausland verzogen und eine Person verstorben. Zieht man diese 61 Personen als qualitätsneutrale Ausfälle ab, verblieb zu Beginn der Feldarbeit eine unbereinigte Bruttostichprobe von 10.863. Darunter waren weitere 13 Kinder und Jugendliche, deren Eltern bei der Basiserhebung eine erneute Kontaktierung abgelehnt hatten, sowie 6 Familien, deren Anschrift nicht mehr ermittelt werden konnte. Für KiGGS Welle 1 werden diese als Nichtteilnehmer gezählt (nicht jedoch als qualitätsneutrale Ausfälle, da aus dem Samp- 


\begin{tabular}{|c|c|c|c|c|}
\hline & Altersjahrgänge in Jahren & Jungen & Mädchen & Gesamt \\
\hline \multirow[t]{7}{*}{ Neue Stichprobe } & 0 & 320 & 314 & 634 \\
\hline & 1 & 337 & 304 & 641 \\
\hline & 2 & 346 & 321 & 667 \\
\hline & 3 & 320 & 281 & 601 \\
\hline & 4 & 335 & 328 & 663 \\
\hline & 5 & 330 & 303 & 633 \\
\hline & 6 & 302 & 314 & 616 \\
\hline \multirow[t]{12}{*}{ Alte Stichprobe } & 7 & 324 & 316 & 640 \\
\hline & 8 & 317 & 341 & 658 \\
\hline & 9 & 336 & 326 & 662 \\
\hline & 10 & 325 & 370 & 695 \\
\hline & 11 & 369 & 346 & 715 \\
\hline & 12 & 404 & 379 & 783 \\
\hline & 13 & 397 & 372 & 769 \\
\hline & 14 & 384 & 399 & 783 \\
\hline & 15 & 384 & 342 & 726 \\
\hline & 16 & 380 & 358 & 738 \\
\hline & 17 & 365 & 379 & 744 \\
\hline & Altersgruppen in Jahren & Jungen & Mädchen & Gesamt \\
\hline \multirow[t]{2}{*}{ Neue Stichprobe } & $0-2$ & 1.003 & 939 & 1942 \\
\hline & $3-6$ & 1.287 & 1.226 & 2513 \\
\hline \multirow[t]{3}{*}{ Alte Stichprobe } & $7-10$ & 1.302 & 1.353 & 2655 \\
\hline & $11-13$ & 1.170 & 1.097 & 2267 \\
\hline & $14-17$ & 1.513 & 1.478 & 2991 \\
\hline Gesamt & 0-17 & 6.275 & 6.093 & 12.368 \\
\hline
\end{tabular}

le Point verzogene Wiedereingeladene im Gegensatz zu Ersteingeladenen - per Definition nicht ausgeschlossen wurden). Letztlich wurden somit 10.844 Kohortenteilnehmende für KiGGS Welle 1 erneut eingeladen (403 mit zusätzlicher Übersetzung).

Insgesamt wurden also 22.678 Einladungsschreiben versendet.

\section{Qualitätsneutrale Ausfälle im Verlauf der Feldarbeit}

Von den 11.834 erstmals eingeladenen 0 - bis 6-Jährigen wurden im Verlauf der Feldarbeit insgesamt 349 (2,9\%) als qualitätsneutrale Ausfälle gewertet. Grund war bei $83,4 \%$ dieser Fälle $(n=291)$, dass das Einladungsschreiben nicht zustellbar war, die Person verzogen war oder dauerhaft außerhalb des Sample Points wohnte. Relativ selten kam vor, dass eine Verständigung aufgrund sprachlicher Probleme unmöglich war $(n=26 ; 7,4 \%)$, dass eine Person doppelt gezogen wurde
( $n=11 ; 3,2 \%$ ) oder zum Erhebungszeitpunkt schon 7 Jahre alt war $(n=21 ; 6,0 \%)$. Für die Ersteingeladenen ergibt sich somit eine bereinigte Bruttostichprobe von 11.485 Personen.

Von den 10.844 wiedereingeladenen Kohortenteilnehmenden wurden im Verlauf der Feldarbeit weitere 8 als qualiins Ausland verzogen, 2 verstorben). Damit ergibt sich für die Wiedereingeladenen eine bereinigte Bruttostichprobe von 10.855 Personen.

\section{Teilnehmende}

Insgesamt nahmen 12.368 Kinder und Jugendliche im Alter von 0 bis 17 Jahren (bzw. deren Eltern) an KiGGS Welle 1 teil (6093 Mädchen, 6275 Jungen; s. • Tab. 2). Die neue Querschnittstichprobe von 0bis 6-Jährigen umfasst 4455 Teilnehmende, die Kohortenstichprobe im Alter von 7 bis 17 Jahren 7913 Teilnehmende. Nur 54 Eltern von Querschnittteilnehmenden tätsneutrale Ausfälle eingestuft (6 waren
(1,2\%) beantworteten den schriftlichen Fragebogen, überwiegend (4401; 98,8\%) entschieden sich die Eltern für das Telefoninterview. Von den 7865 teilnehmenden Eltern der Kohortenteilnehmenden wurde überwiegend das Telefoninterview absolviert $(n=7716,98,1 \%)$ und nur selten $(n=149 ; 1,9 \%)$ ein schriftlicher Fragebogen beantwortet. Bei den 5258 Teilnehmenden in der Altersgruppe 11 bis 17 Jahre nahmen überwiegend sowohl die Eltern als auch die Kinder und Jugendlichen an der Befragung teil ( $n=4907,93,3 \%)$, selten nur die Eltern $(n=303,5,8 \%)$ oder nur die Kinder und Jugendlichen $(n=48$; $0,9 \%)$.

\section{Response}

In KiGGS Welle 1 lag die Teilnahmequote bei den erstmals Eingeladenen bei $38,8 \%$, bei den Wiedereingeladenen bei $72,9 \%$. Altersbezogene Unterschiede zeigten sich bei den Ersteingeladenen nicht ( 0 bis 2 Jahre 39,1\%; 3 bis 6 Jahre 38,6\%) und bei den Wiedereingeladenen nur in geringem Ausmaß (7 bis 10 Jahre 70,9\%; 11 bis 13 Jahre $76,0 \%$; 14 bis 17 Jahre 72,4\%).

\section{Gründe für Nichtteilnahme}

Die einzelnen Gründe der Nichtteilnahme kommen bei Erst- und Wiedereingeladenen zumeist gleich anteilig vor (die folgenden Prozentangaben beziehen sich jeweils auf die bereinigte Bruttostichprobe, d. h. auf alle Eingeladenen abzüglich der qualitätsneutralen Ausfälle).

Sowohl bei Erst- als auch bei Wiedereingeladenen ist der Anteil mit expliziter Ablehnung der Studienteilnahme gering (4,4 und 4,5\%). Weitere jeweils 5,2\% verschoben die vereinbarten Termine einoder mehrmals ohne Ergebnis oder sandten die Fragebögen nicht zurück, was als implizite Ablehnung zu werten ist. 1,7 bzw. 1,8\% der Interviews konnten wegen Abbruchs oder fehlender Einwilligungserklärung nicht verwertet werden.

Dagegen konnten relativ viele Familien nicht erreicht werden, insbesondere bei den Ersteingeladenen: Hier war fast die Hälfte $(47,9 \%)$ telefonisch nicht erreichbar - in 90,6\% dieser Fälle, weil keine Telefonnummer ermittelt werden konnte. Bei den Wiedereingeladenen lag 
der Anteil mit 15,4\% deutlich niedriger, weil hier in der Regel Telefonnummern aus der Basiserhebung vorlagen.

\section{Nonresponse-Bias, Gewichtung und "Repräsentativität ${ }^{\prime \prime}$}

Um bei den 7- bis 17-jährigen Kohortenteilnehmenden den möglichen Selektionsbias und dessen Auswirkungen auf die Prävalenzschätzer von Gesundheitsindikatoren in KiGGS Welle 1 zu untersuchen, wurde verglichen, inwieweit sich die Prävalenz ausgewählter Merkmale der KiGGS-Basiserhebung zwischen den Teilnehmenden und Nichtteilnehmenden an KiGGS Welle 1 unterscheiden (• Tab. 3). Die Spalte D der • Tab. 3 zeigt deskriptiv, dass kaum Unterschiede bei der Wiederteilnahmebereitschaft in Abhängigkeit von einzelnen Gesundheitsparametern existieren. So haben beispielsweise Kinder und Jugendliche mit Asthmaerkrankung zum Erhebungszeitpunkt der KiGGS-Basiserhebung in der Wiederbefragung ähnlich der Gesamtresponse mit $72,5 \%$ teilgenommen, sodass es zu keiner Selektivität aufgrund der Erkrankung gekommen ist. Ähnliche Ergebnisse sind auch bei den meisten weiteren Parametern zu beobachten. Bei den hier dargestellten Gesundheitsindikatoren zeigt sich nur bei Kindern und Jugendlichen, die im Hinblick auf psychische Auffälligkeiten bei den 4 Problemskalen des Strengths and Difficulties Questionnaires (SDQ) einen auffälligen Gesamtproblemwert (vgl. [14]) aufweisen, die wenig Sport treiben oder die bei alleinerziehenden Müttern wohnen, eine unterdurchschnittliche Wiederteilnahmebereitschaft. Hinsichtlich der soziodemografischen Parameter (Sozialstatus bzw. Migrationshintergrund) sind die Unterschiede am größten.

Obwohl die Prävalenzunterschiede zwischen Wiederteilnehmern (Spalte E) und Nicht-Wiederteilnehmern (• Tab. 3 , Spalte F) oftmals fallzahlbedingt statistische Signifikanz erreicht, ist der errechnete Nonresponse-Bias (• Tab. 3, Spalte H) überwiegend sehr niedrig. So beträgt zum Beispiel der Nonresponse-Bias bei der Variable "wichtigster Versicherungsschutz" nur - 0,9\%, obwohl der Anteil der Mitglieder in der gesetzlichen Krankenversi- cherung (GKV-Versicherte) bei den Wiederteilnehmern mit 87,8\% $(86,6-88,9)$ signifikant niedriger als bei den Nicht-Wiederteilnehmern (91,0\%, 89,7-92,2) ist ( $p$ Wert $<0,001)$. Da der Bias sowohl vom Unterschied zwischen Teilnehmenden und Nicht-Teilnehmenden als auch von der Nonresponse abhängt, ist aufgrund der niedrigen Nonresponse $(27,8 \%)$ die Schätzung des Anteils der GKV-Versicherten nur auf der Basis der Wiederteilnehmerstichprobe (• Tab. 3, Spalte E) nahe dem tatsächlichen Anteil von 88,8\% (- Tab. 3, Spalte G). Einen etwas höheren Nonresponse-Bias weisen die Variablen „SDQ-Gesamtproblemwert“, „Schwimmen/Turnen/Sport“-Tätigkeit, „Rauchen in der Wohnung“ und „Hauptaufenthaltsort" bei der Mutter sowie Migrationshintergrund und niedriger sozialer Status auf.

Die gezeigten Wiederteilnahmeunterschiede wurden durch ein multivariates logistisches Regressionsmodell überprüft. Hierbei wurden alle verfügbaren Informationen sowohl hinsichtlich Soziodemografie und Teilnehmendengewinnungsprozess als auch hinsichtlich gesundheits- und verhaltensbezogener Erhebungsdaten einbezogen. Das Modell diente auch dazu, die Nonresponse bzw. die Selektivität bei der Berechnung der Gewichte zu berücksichtigen und damit den oben gezeigten potenziellen Bias bei der Schätzung von Prävalenzen zu korrigieren. In • Tab. 4 werden die signifikanten Prädiktoren des logistischen Regressionsmodells zur Bestimmung der Wiederteilnahmewahrscheinlichkeit für die 7- bis 17-Jährigen wiedergegeben.

Das logistische Regressionsmodell zeigte, dass vor allem das Alter der Mutter (OR: 0,50, $95 \%$-KI: 0,38-0,65 für „<25 Jahre“ bzw. OR: 0,65, $95 \%$-KI: 0,56-0,75 für „25-29 Jahre“ vs. „30 Jahre und älter"), der Rauchstatus der Mutter (OR: 0,68, 95\%-KI: 0,61-0,75 für „Rauchen Ja"), die Familiensituation (OR: 1,42, $95 \%$-KI: $1,25-1,62$ für „Kind/Jugendlicher lebt bei beiden leiblichen Eltern"), der Migrationshintergrund des Kindes/Jugendlichen (OR: 0,72, 95\%-KI: 0,63-0,82 für „Migration Ja“) sowie die Teilnahmebereitschaft an den Modulstudien der KiGGS-Basiserhebung (z. B.: OR: 0,57, $95 \%$-KI: 0,50-0,65 für „keine Teil- nahme an der zusätzlichen Modulstudie Motorik“") die stärksten Einflussfaktoren auf die Bereitschaft zur Wiederteilnahme waren. Weitere signifikante Prädiktoren für eine niedrige Teilnahmebereitschaft waren eine niedrige Schul-/Berufsausbildung und Berufsstellung der Mutter sowie das Familieneinkommen (- Tab.4).

In - Tab. 3 zeigt Spalte I die Prävalenzschätzung verschiedener Gesundheitsparameter der KiGGS-Basiserhebung bei Wiederteilnehmenden unter Berücksichtigung des Wiederteilnahmegewichtes. Im Vergleich mit der Spalte G aus - Tab. 3 wird die Reduzierung des Nonresponse-Bias und damit der Verzerrung bei der Prävalenzschätzung deutlich. Bei fast allen der untersuchten Parameter beträgt die Differenz zu den tatsächlichen Prävalenzen auf Basis der Gesamtstichprobe der KiGGS-Basiserhebung weniger als einen Prozentpunkt. Die Berücksichtigung der Wiederteilnahmewahrscheinlichkeit bei der Berechnung der Gewichte verbessert vor allem die Prävalenzschätzungen bei solchen Indikatoren, die vor der Gewichtung einen höheren Nonresponse-Bias aufwiesen (Sportaktivität, Rauchen in der Wohnung, SDQGesamtproblemwert, Hauptaufenthaltsort). Auch der Unterschied zwischen geschätztem und tatsächlichem Anteil in den soziodemografischen Variablen „Migrationshintergrund“ und "Sozialstatus“ ist nicht mehr vorhanden.

Definiert man Repräsentativität als strukturelle Übereinstimmung einer Stichprobe mit der Grundgesamtheit im Hinblick auf die Verteilung ausgewählter Merkmale, so zeigt $\bullet$ Tab. 5, dass nach Anpassungsgewichtung eine sehr gute Übereinstimmung für die soziodemografischen Merkmale Altersgruppe, Geschlecht, höchste Bildung des Haushaltsvorstands sowie den Regionalstraten Ost, West und Berlin besteht. Die Anpassungsgewichtung gleicht insbesondere die vergleichsweise geringe Beteiligung der unteren Bildungsgruppe sowie das designbedingte Oversampling der ostdeutschen Bundesländer aus. Gesundheitsbezogene Merkmale von Kindern und Jugendlichen, die einen Vergleich zwischen der Bevölkerung Deutschlands und der Studienpopulation von KiGGS Welle 1 erlauben, sind rar. So entspricht der Anteil 


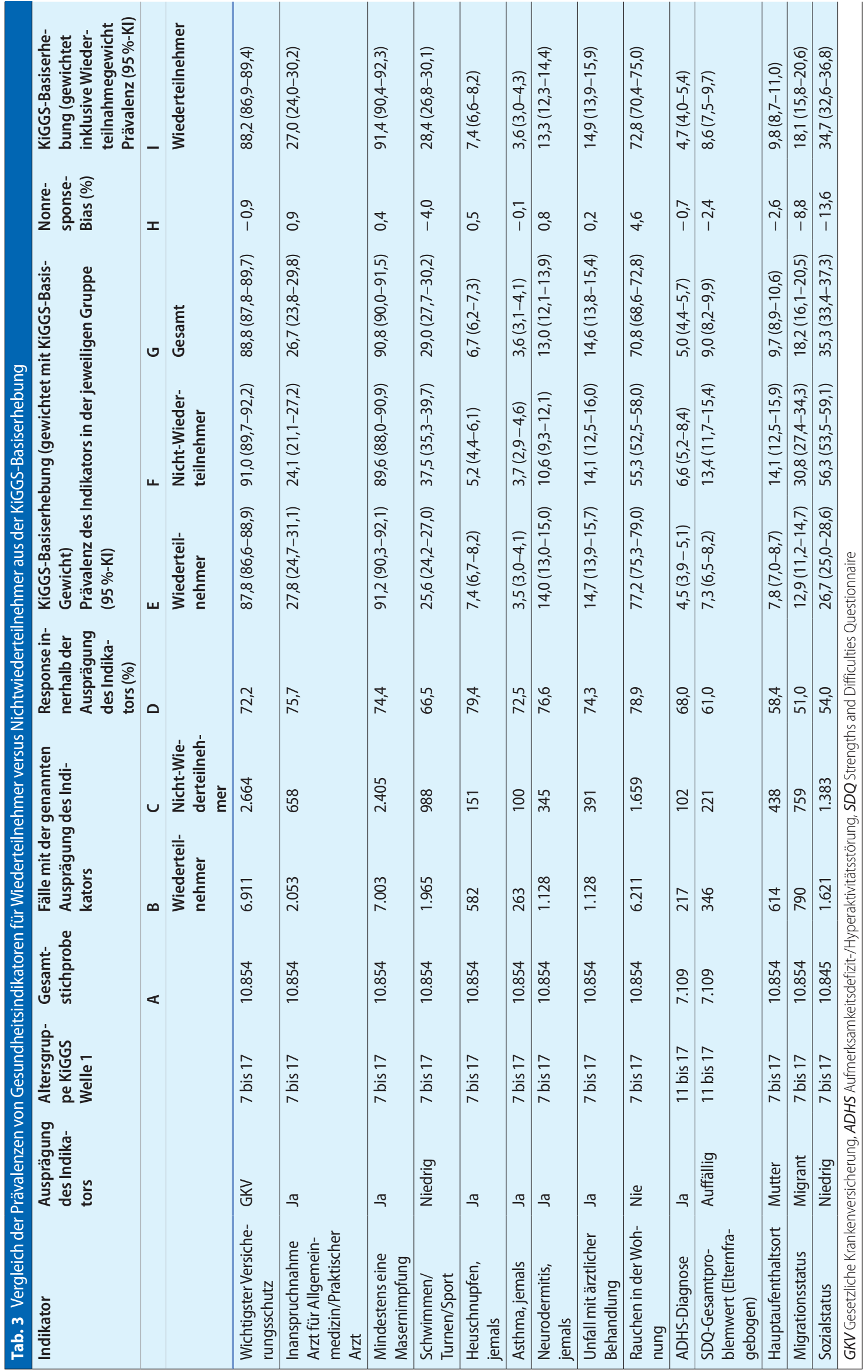




\section{Leitthema}

Tab. 4 Signifikante Prädiktoren im Wiederteilnahme-Modell

\begin{tabular}{|c|c|c|}
\hline Variablen & Effekt & Odds-Ratio (95\%-KI) \\
\hline \multirow{10}{*}{ Alter des Kindes in KiGGS Welle 1} & 7 vs. 17 Jahre & $1,358(1,064-1,732)$ \\
\hline & 8 vs. 17 Jahre & $1,184(0,937-1,497)$ \\
\hline & 9 vs. 17 Jahre & $1,419(1,126-1,787)$ \\
\hline & 10 vs. 17 Jahre & $0,989(0,788-1,242)$ \\
\hline & 11 vs. 17 Jahre & $1,255(0,998-1,579)$ \\
\hline & 12 vs. 17 Jahre & $1,459(1,16-1,836)$ \\
\hline & 13 vs. 17 Jahre & $1,363(1,092-1,702)$ \\
\hline & 14 vs. 17 Jahre & $1,229(0,986-1,531)$ \\
\hline & 15 vs. 17 Jahre & $1,063(0,854-1,322)$ \\
\hline & 16 vs. 17 Jahre & $1,081(0,87-1,344)$ \\
\hline Geschlecht & Junge vs. Mädchen & $0,964(0,876-1,061)$ \\
\hline \multicolumn{3}{|c|}{ Familiäre Variablen (KiGGS-Basiserhebung) } \\
\hline $\begin{array}{l}\text { Migrationshintergrund des } \\
\text { Kindes }\end{array}$ & Ja/keine Angabe vs. nein & $0,719(0,628-0,822)$ \\
\hline \multirow[t]{2}{*}{ Alter der Mutter } & 17 bis 24 Jahre vs. 30 Jahre oder älter & $0,498(0,383-0,646)$ \\
\hline & 25 bis 29 Jahre vs. 30 Jahre oder älter & $0,651(0,563-0,752)$ \\
\hline Schulabschluss der Mutter & $\begin{array}{l}\text { Hauptschule/Sonstiges vs. Realschu- } \\
\text { le/Abitur }\end{array}$ & $0,702(0,625-0,789)$ \\
\hline Rauchen der Mutter & Ja/keine Angabe vs. nein & $0,679(0,612-0,752)$ \\
\hline \multirow{2}{*}{$\begin{array}{l}\text { ISCED-Bildungsgruppe } \\
\text { (Maximum der Eltern im HH) }\end{array}$} & Niedrig/keine Angabe vs. hoch & $0,653(0,522-0,816)$ \\
\hline & Mittel vs. hoch & $0,774(0,681-0,879)$ \\
\hline Kind/Jugendlicher lebt bei ... & $\begin{array}{l}\text { Beiden leiblichen Eltern vs. andere } \\
\text { Konstellationen }\end{array}$ & $1,419(1,245-1,619)$ \\
\hline $\begin{array}{l}\text { Arbeitslosigkeit des Haushalts- } \\
\text { vorstandes }\end{array}$ & Ja/keine Angabe vs. nein & $0,659(0,563-0,771)$ \\
\hline \multirow{2}{*}{$\begin{array}{l}\text { Winklerscore }{ }^{\text {a: }} \text { Schul- und Berufs- } \\
\text { ausbildung der Mutter }\end{array}$} & 1/keine Angabe vs. 5-7 & $0,682(0,553-0,84)$ \\
\hline & $2-4$ vs. $5-7$ & $0,87(0,743-1,018)$ \\
\hline $\begin{array}{l}\text { Berufsstatus (Autonomie n. Hoff- } \\
\text { meyer-Zlotnik) }\end{array}$ & $\begin{array}{l}\text { Niedrig/mäßig/keine Angabe vs. } \\
\text { mittel/gehoben/hoch }\end{array}$ & $0,879(0,771-1,001)$ \\
\hline \multirow{2}{*}{$\begin{array}{l}\text { Winklerscore }{ }^{\mathrm{a}} \text { : Berufliche } \\
\text { Stellung der Mutter }\end{array}$} & 1/keine Angabe vs. 4-7 & $0,677(0,578-0,793)$ \\
\hline & $2-3$ vs. $4-7$ & $0,768(0,661-0,891)$ \\
\hline Monatliches Familieneinkommen & $\begin{array}{l}<1750 \text { EUR/keine Angabe } \\
\text { vs. } \geq 1750 \text { EUR }\end{array}$ & $0,739(0,662-0,826)$ \\
\hline \multicolumn{3}{|c|}{ Beteiligungsvariablen (KiGGS-Basiserhebung) } \\
\hline $\begin{array}{l}\text { Ernährungsfragebogen beant- } \\
\text { wortet }\end{array}$ & Nein vs. ja & $0,615(0,506-0,746)$ \\
\hline $\begin{array}{l}\text { Teilnahme an der Modulstudie } \\
\text { Kinder-Umwelt-Survey }\end{array}$ & Nein/trifft nicht zu vs. ja & $0,692(0,589-0,813)$ \\
\hline $\begin{array}{l}\text { Teilnahme an der Modulstudie } \\
\text { Motorik }\end{array}$ & Nein/trifft nicht zu vs. ja & $0,574(0,504-0,653)$ \\
\hline \multirow[t]{2}{*}{ Blutprobe abgegeben } & Kein Blut und kein Urin vs. nur Urin & $0,764(0,584-1)$ \\
\hline & Blut (mit/ohne Urin) vs. nur Urin & $1,232(1,081-1,403)$ \\
\hline $\begin{array}{l}\text { Ärztliches Interview (CAPI) } \\
\text { geführt mit ... }\end{array}$ & $\begin{array}{l}\text { Mutter/andere/keine Angabe vs. } \\
\text { Vater }\end{array}$ & $1,286(1,129-1,464)$ \\
\hline Untersucherin in der Feldarbeit & Gruppe A vs. Gruppe B & $0,589(0,454-0,763)$ \\
\hline \multicolumn{3}{|c|}{ Regionale Variablen (KiGGS-Basiserhebung) } \\
\hline Bundesland & 1 vs. $2^{\text {b }}$ & $0,737(0,662-0,82)$ \\
\hline BIK-Klassifikation & 1 vs. $2^{c}$ & $1,164(1,05-1,29)$ \\
\hline \multicolumn{3}{|c|}{$\begin{array}{l}\text { HH Haushalt, ISCED International Standard Classification of Education } \\
\text { aje mehr Punkte (max. 7), desto höher ist der Stand der Berufsausbildung bzw. die berufliche Stellung } \\
\text { b1 =Schleswig-Holstein, Hamburg, Hessen, Rheinland-Pfalz, Saarland, Berlin, Sachsen-Anhalt, Thüringen; } \\
\text { 2= Niedersachsen, Bremen, Nordrhein-Westfalen, Baden-Württemberg, Bayern, Brandenburg, Mecklenburg- } \\
\text { Vorpommern, Sachsen } \\
{ }^{1} 1 \leq<50.000 \text { Einwohner, Kernstadt 50.000-100.000 Einwohner, Umland 100.000 Einwohner und mehr; } \\
2=\text { Umland 50.000-100.000 Einwohner, Kernstadt 100.000 Einwohner und mehr }\end{array}$} \\
\hline
\end{tabular}

der Kinder und Jugendlichen mit einer amtlich anerkannten Schwerbehinderung von $1 \%$ in KiGGS Welle 1 ziemlich genau dem Anteil von 1,05\% laut amtlicher Statistik [15]. Ein weiteres Beispiel betrifft den Anteil der Kinder und Jugendlichen, die gesetzlich krankenversichert sind. Dieser Anteil beträgt unter den Survey-Teilnehmenden knapp $89 \%$ und zeigt damit eine gute Übereinstimmung mit den tatsächlichen $87 \%$ in der entsprechenden Teilbevölkerung laut amtlicher Statistik [16, 17].

\section{Diskussion}

Ein Ziel von KiGGS Welle 1 war es, 6 Jahre nach der KiGGS-Basiserhebung erneut aktuelle populationsbezogene Befragungsdaten zu erheben, die Aussagen über die Wohnbevölkerung der 0bis 17-Jährigen in Deutschland zulassen. Das Stichprobendesign sah die Kombination einer neu gezogenen Stichprobe (0 bis 6 Jahre) mit der vorhandenen und wiedereingeladenen Stichprobe der Teilnehmenden der Basiserhebung (inzwischen 7 bis 17 Jahre) vor. Solche Mischdesigns werden in verschiedenen bevölkerungsbezogenen sozial- und wirtschaftswissenschaftlichen sowie epidemiologischen Studien mit vergleichbaren Zielsetzungen eingesetzt. In die Sozialberichterstattung des Bundes und der Europäischen Union fließen beispielsweise sowohl Längsschnittanalysen als auch Prävalenzschätzungen und Trendanalysen des Sozio-oekonomischen Panels (SOEP) und der Studie der Europäischen Union über Einkommen und Lebensbedingungen (EU-SILC) ein $[18,19]$. Aus dem Bereich der Epidemiologie und der Gesundheitsberichterstattung ist - neben Gesundheitssurveys mit gemischten Querschnitt- und Längsschnittstichproben in Norwegen [20], Finnland [20], Australien [21] und Neuseeland [22] - insbesondere die National Longitudinal Study of Adolescence Health (AddHealth) aus den USA zu nennen, die unter anderem Prävalenzschätzungen und Trendentwicklungen aus Längsschnittdaten für wichtige Gesundheits- und Verhaltensindikatoren der Jugendlichen und jungen Erwachsenen in den USA liefert [23-28]. 


\begin{tabular}{|c|c|c|c|}
\hline \multirow[t]{2}{*}{ Variable } & \multicolumn{3}{|l|}{ Anteile in \% } \\
\hline & Ungewichtet & Gewichtet & Bevölkerung \\
\hline \multicolumn{4}{|l|}{ Alter männlich ${ }^{a}$} \\
\hline 0 bis 2 Jahre & 16,0 & 14,3 & 14,4 \\
\hline 3 bis 6 Jahre & 20,5 & 20,9 & 21,0 \\
\hline 7 bis 10 Jahre & 20,8 & 22,3 & 22,3 \\
\hline 11 bis 13 Jahre & 18,7 & 18,1 & 18,1 \\
\hline 14 bis 17 Jahre & 24,1 & 24,3 & 24,3 \\
\hline \multicolumn{4}{|l|}{ Alter weiblich $^{\mathrm{a}}$} \\
\hline 0 bis 2 Jahre & 15,4 & 14,4 & 14,4 \\
\hline 3 bis 6 Jahre & 20,1 & 20,9 & 21,0 \\
\hline 7 bis 10 Jahre & 22,2 & 22,4 & 22,3 \\
\hline 11 bis 13 Jahre & 18,0 & 18,1 & 18,1 \\
\hline 14 bis 17 Jahre & 24,3 & 24,3 & 24,2 \\
\hline \multicolumn{4}{|c|}{ Höchste Bildung des Haushaltsvorstands (Casmin) ${ }^{b}$} \\
\hline Einfache Bildung & 8,1 & 28,0 & 28,0 \\
\hline Mittlere Bildung & 56,6 & 46,5 & 46,9 \\
\hline Höhere Bildung & 35,4 & 25,5 & 25,2 \\
\hline \multicolumn{4}{|l|}{ Straten $^{\mathrm{a}}$} \\
\hline West & 67,2 & 83,9 & 83,7 \\
\hline Ost & 30,2 & 12,6 & 12,6 \\
\hline Berlin & 2,5 & 3,5 & 3,8 \\
\hline \multicolumn{4}{|l|}{ Krankenversicherung des Kindes ${ }^{c}$} \\
\hline Gesetzliche Krankenversicherung & 86,0 & 88,6 & 87,0 \\
\hline \multicolumn{4}{|l|}{ Behinderung $^{d}$} \\
\hline $\begin{array}{l}\text { Amtlich anerkannte Schwerbehin- } \\
\text { derung }\end{array}$ & 0,97 & 1,00 & 1,05 \\
\hline \multicolumn{4}{|l|}{$\begin{array}{l}\text { aStatistisches Bundesamt (31.12.2010) } \\
\text { bMikrozensus } 2009 \\
\text { CBundesministerium für Gesundheit } \\
\text { dMikrozensus } 2010\end{array}$} \\
\hline
\end{tabular}

Während der große Vorteil von gemischten Designs darin besteht, gleichzeitig Querschnitts-, Trend- und Lebensverlaufsanalysen zu ermöglichen, gibt es - vor allem im Hinblick auf Selektionseffekte und Gewichtung - auch Nachteile, die für KiGGS nachfolgend diskutiert werden.

Eine Voraussetzung für eine qualitativ hochwertige bevölkerungsbezogene Bruttostichprobe ist, dass sie eine $\mathrm{Zu}$ fallsauswahl ist [29], d. h. keine Bevölkerungsgruppe systematisch bei der Stichprobenziehung ausgeschlossen wird. In der KiGGS-Basiserhebung kam deshalb ein komplexes mehrstufiges Stichprobenverfahren zur Anwendung. Die Auswahl der 167 Untersuchungsorte erfolgte in der Basiserhebung nach einem sorgfältig geplanten, mehrfach geschichteten Zufallsverfahren. Dadurch konnte sicher- gestellt werden, dass bei der Auswahl der Orte die wesentlichen räumlichen und strukturellen Merkmale Deutschlands bestmöglich abgebildet werden. In jedem der 167 Untersuchungsorte erfolgte die Personenauswahl in der Basiserhebung durch eine uneingeschränkte Zufallsauswahl aus dem Einwohnermelderegister. Die Ziehung für die neue Stichprobe der 0 - bis 6-Jährigen erfolgte in KiGGS Welle 1 in denselben Sample Points nach gleicher Systematik.

Für die Längsschnittteilnehmenden stellt die Beibehaltung der Sample Points kein grundlegendes Problem dar. Die ausgewählten Sample Points bildeten für den Erhebungszeitraum der Basiserhebung die Bevölkerung der Kinder und Jugendlichen nach Gemeindegröße, Bundesland und BIK-Klassifikation im Erwartungswert unverzerrt ab. Da die Durchführung des Telefoninterviews bei Welle 1 nicht an den ehemaligen Wohnort gebunden war und alle Teilnehmenden der Basiserhebung eingeladen wurden, ist davon auszugehen, dass auch Wanderungsbewegungen innerhalb Deutschlands von der Studienpopulation abgebildet werden. Dies gilt allerdings nicht für Zuwanderungen aus dem Ausland nach Deutschland, sodass Kinder, Jugendliche und junge Volljährige, die zum Zeitpunkt der KiGGSBasiserhebung nicht in Deutschland lebten, auch in KiGGS Welle 1 nicht enthalten sind. Für die Querschnittstichprobe stellt sich die Frage, inwiefern die Sample Points der Basiserhebung angesichts möglicher Veränderungen der Bevölkerungsstruktur beibehalten werden können. Die Verteilung der Kinder und Jugendlichen auf die Bundesländer ist allerdings nahezu gleich geblieben, wenn man die für die Ziehung der Sample Points verwendeten Bevölkerungszahlen mit den Zahlen aus der (aktuelleren) BIK2009Klassifikation [30] vergleicht, die ggf. für die Ziehung neuer Sample Points für Welle 1 maßgeblich gewesen wäre. Die Verteilung der Gemeindestruktur hat sich dagegen verändert, nicht zuletzt aufgrund von Gebietsreformen und Überarbeitungen der BIK-Klassifikation. Daran gemessen hätten bei einer Neuziehung ca. $10 \%$ der Sample Points aus einer anderen BIKKlasse gezogen werden müssen. Dieser Anteil ist jedoch nicht so groß, dass stärkere Verzerrungen zu erwarten sind. Zudem sind die Veränderungen auf Bevölkerungsebene vermutlich stärker durch administrative Prozesse (Gemeindereformen und neue BIK-Klassifizierung) bedingt als durch Wanderungsbewegungen.

Eine weitere Voraussetzung für eine qualitativ hochwertige Nettostichprobe ist die Reduzierung von Selektionseffekten bzw. deren Berücksichtigung und teilweise Korrektur über die Gewichtung der Daten [12]. Wie alle empirischen Untersuchungen an Freiwilligen unterliegen auch die KiGGS-Erhebungen einem gewissen Selbstselektionseffekt. Dabei zeigen Bevölkerungsgruppen mit höherer Schulbildung und höherem Gesundheitsbewusstsein in der Regel eine höhere Teilnahmebereitschaft als andere Bevölkerungsteile. Da die meisten gesundheitsbezogenen Merkmale Zusammenhänge 
zum Bildungsgrad aufweisen, kann dies zu einem Selektionsbias führen mit möglicher Verzerrung der Prävalenzschätzer für diese Merkmale.

KiGGS Welle 1 besteht zudem - wie bereits dargestellt - aus 2 verschiedenen Stichproben. Während die Querschnittstichprobe bei einer relativ niedrigen Teilnahmequote $(38,8 \%)$ einer nur einmaligen Selbstselektion unterlag, fand in der wiederbefragten Kohortenstichprobe eine zweimalige Selbstselektion statt, allerdings bei insgesamt bedeutend höheren Teilnahmequoten (72,9\%). Dass auch die Wiederteilnahmebereitschaft der Kohortenteilnehmenden Zusammenhänge zu gesundheitsbezogenen Merkmalen aufweist, wurde im Ergebnisteil dieses Beitrags beschrieben. Durch die Wiederteilnahmegewichtung kann diese Selektion jedoch besser ausgeglichen werden als bei den Querschnittprobanden, da mehr Variablen zur Gewichtung zur Verfügung stehen.

Im Vergleich zur KiGGS-Basiserhebung, bei der für den Altersbereich 0 bis 6 Jahre eine Response von $66 \%$ [5] erreicht wurde, fällt die Teilnahmequote bei den Ersteingeladenen von KiGGS Welle 1 mit rund $39 \%$ deutlich niedriger aus. Ein Vergleich der Response mit den Ergebnissen anderer Studien ist schwierig, da es kaum bevölkerungsbezogene Studien für diesen Altersbereich gibt. Bei der zeitlich parallel durchgeführten RKIErwachsenengesundheitsstudie DEGS1 wurde bei Ersteingeladenen eine ähnliche Response von $42 \%$ erreicht; jedoch handelte es sich um einen Untersuchungssurvey und einen anderen Altersbereich (18 bis 79 Jahre) [31]. Telefonische Erhebungen ohne vorherige schriftliche Kontaktaufnahme im Rahmen des Gesundheitsmonitorings des Robert Koch-Instituts (GEDA-Studie) weisen eine Response um die $29 \%$ aus [32]. Häder [33] und Engel et al. [34] fassen die in den letzten Jahren entwickelten und empirisch überprüften theoretischen Ansätze zur Erklärung der Teilnahmebereitschaft bzw. Nonresponse zusammen. Die wichtigsten Erklärungsversuche stellen die Teilnahme als eine rationale Entscheidung [35], als heuristische Entscheidung [36], als Ergebnis eines sozialen Austauschs [37] oder als Ergebnis eines geplanten Verhaltens [38] dar. Da- rüber hinaus kann nach Groves/Couper [39] die Teilnahmeentscheidung als Ergebnis von 3 Wahrscheinlichkeiten - die Zielperson zu lokalisieren, Kontakt aufzunehmen und die Zielperson zu überzeugen - gesehen werden [34]. Der niedrige Anteil der qualitätsneutralen Ausfälle in der KiGGS Welle 1 zeugt von einer hohen Wahrscheinlichkeit, die eingeladenen Familien zu lokalisieren. Die geringere Response der neuen Stichprobe erklärt sich größtenteils daraus, dass im Gegensatz zur KiGGS-Basiserhebung aus Kostengründen keine Hausbesuche zwecks Teilnehmendengewinnung durchgeführt werden konnten. Hinzu kommt, dass Rufnummern zur telefonischen Teilnehmendenwerbung bei rund $43 \%$ nicht ermittelbar waren mit der Folge stark eingeschränkter Ansprache- und Erinnerungsmöglichkeiten und damit einem hohen Anteil von nie erreichten Personen. Schließlich war eine explizite Ablehnung nur bei etwa $4 \%$ das Ergebnis der Teilnehmergewinnung.

Eine hohe Nonresponse geht jedoch nicht zwangsläufig mit Einbußen in der Stichprobenqualität einher, ebenso stellt eine hohe Teilnahmequote kein hinreichendes Gütekriterium für die Qualität einer Stichprobe dar. Ein Nonresponse-Bias entsteht - unabhängig von der Höhe der Response - nur dann, wenn sich Teilnehmende und Nichtteilnehmende bzw. Teilnehmende und Grundgesamtheit in den zu schätzenden Gesundheitsparametern deutlich unterscheiden, d. h., wenn die zu erhebenden Parameter mit der Teilnahmebereitschaft assoziiert sind [40]. Zur Einschätzung der Selektivitätseffekte in KiGGS Welle 1 wurden deshalb neben der Response auch weitere, in der Fachliteratur empfohlene Ansätze verfolgt [12, 41]. Insbesondere für den Altersbereich der 7bis 17-Jährigen - also für die KiGGS-Kohorte - wurden die indikatorenspezifische Response und der potenzielle Nonresponse-Bias für einzelne in der Basiserhebung erhobene Zielvariablen berechnet. Die Auswahl der in diesem Artikel univariat-deskriptiv berichteten Indikatoren orientiert sich thematisch an den in diesem Sonderheft publizierten Themen. Nur bei einzelnen Indikatoren bestand ein Unterschied zwischen Teilnehmenden und Nicht-Teilnehmenden bzw. ein Zusam- menhang zwischen Gesundheitsindikator und Teilnahmebereitschaft.

Eine Möglichkeit, die beschriebene Nonresponse und Selektion zumindest teilweise zu kompensieren, bieten Gewichtungen. Abweichungen der Verteilung wichtiger Grundcharakteristika in der Studienpopulation von der Grundgesamtheit werden üblicherweise durch Gewichtungsfaktoren, bestehend aus Design- und Anpassungsgewicht, ausgeglichen. In KiGGS Welle 1 wurde darüber hinaus bei der Gewichtung der Kohortenteilnehmenden die ungleich hohe Wiederteilnahmebereitschaft mit berücksichtigt. Die bei der KiGGS-Basiserhebung gewonnenen Einflussgrößen waren hier weniger die Gesundheitsindikatoren selbst als vielmehr soziodemografische und auch Verhaltensvariablen. Im Ergebnis entsprechen die Verteilungen der Merkmale Alter, Bildung, Ost/ West, Krankenversicherungsstatus und Anteil mit anerkannter Schwerbehinderung weitgehend den aus amtlichen Statistiken zugänglichen Verteilungen in der Gesamtbevölkerung.

Die Verwendung von Anpassungsgewichtungen ist nicht unumstritten, wird jedoch in vielen großen bevölkerungsbezogenen Studien eingesetzt [beispielsweise National Health and Nutrition Examination Survey (NHANES) [42], German Longitudinal Election Study (GLES) [43], SOEP [44]]. Als Voraussetzung für den Einsatz von Anpassungsgewichten nennt Häder [33] zum einen, dass die Verteilung der Gewichtungsvariable in der Bevölkerung bekannt sein muss. Zum anderen müssen die Gewichtungsvariablen mit den interessierenden Zielvariablen korrelieren [33, 45, 46], und die Verzerrung darf nicht zu stark sein, damit keine unrealistisch hohen Gewichte entstehen [33]. Auch Wiederteilnahmegewichte zum Ausgleich der longitudinalen Ausfälle werden in vielen Studien verwendet [SOEP [44], GLES [43], Swiss Household Panel [47], European Community Household Panel (ECHP) [48]]. Diese Methoden sind auch in der statistischen Literatur ausführlich beschrieben [49-52]. Allen Methoden ist gemeinsam, dass sie nur dann komplett unverzerrte Schätzer liefern können, wenn die Zielvariablen „missing at random“ (MAR) in der Ter- 
minologie von Little und Rubin [53] sind, d. h., wenn die Non-Response bzw. die longitudinalen Ausfälle komplett durch die Variablen erklärt werden können, die in die Gewichtung eingehen [45, 48, 54]. Dieser Idealzustand ist in der Praxis sicher nur selten vollständig zu erreichen. Es kann daher, wie in jeder epidemiologischen Studie, in KiGGS nicht ausgeschlossen werden, dass sich die Studienpopulation in gewissen, nicht messbaren Merkmalen von der Bevölkerung unterscheidet, mit entsprechenden Einschränkungen der externen Validität. So ist es etwa möglich, dass in der Gruppe mit einfacher Bildung nicht-teilnehmende Eltern eher ein ungünstigeres Gesundheitsverhalten aufweisen als teilnehmende Eltern. Dieser Unterschied bewirkt möglicherweise eine Verzerrung der Prävalenzschätzer, die sich auch durch eine Hochgewichtung der einfachen Bildungsgruppe aufgrund ihrer niedrigeren Teilnahmerate nicht vollständig ausgleichen lässt. $\mathrm{Da}$ sich jedoch auch bei den teilnehmenden Eltern für viele Zielvariablen ein Zusammenhang zum Bildungsstand der Eltern zeigt, wobei in der Regel die höheren Bildungsgruppen die günstigeren Ausprägungen aufweisen, kann man davon ausgehen, dass die nach Bildung gewichteten Prävalenzschätzer eine geringere Verzerrung aufweisen als ungewichtete Prävalenzschätzer. Dies ist jeweils im Kontext der konkreten thematischen Auswertung $\mathrm{zu}$ diskutieren. Einige Autoren weisen darauf hin, dass gewichtete Schätzer stärker verzerrt sein können als ungewichtete $[45,54,55]$. Simulationsstudien deuten jedoch darauf hin, dass diese Situation nur bei starker Fehlspezifikation der Gewichte eintritt $[55,56]$. Anpassungsgewichtungen werden auch dafür kritisiert, dass sie die Varianz der Schätzer erhöhen [54]. Dem ist entgegenzuhalten, dass eine niedrige Varianz per se kein Qualitätskriterium für einen Schätzer sein kann, falls dieser nicht unverzerrt ist. Eine höhere Varianz mit daraus resultierenden breiteren Konfidenzintervallen kann vielmehr die den Daten bedingt durch die Non-Response innewohnende Unsicherheit auch quantitativ ausdrücken und bei der Auswertung vor falschen Schlüssen aufgrund falsch positiver statistisch signifikanter Ergebnisse schützen.
Durch die Verwendung von 2 verschiedenen Stichproben in KiGGS Welle 1 entsteht, unabhängig vom Erfolg der Gewichtung, ein methodischer Bruch, dessen mögliche Auswirkungen auf die Prävalenzschätzer der Altersgruppen 0 bis 6 Jahre bzw. den älteren Teilnehmenden im jeweiligen inhaltlichen Kontext von Auswertungen zu diskutieren ist.

Die Befragungen erfolgten in der KiGGS-Basiserhebung zum kleineren Teil durch ein standardisiertes ärztliches Interview mit dem begleitenden Elternteil (Krankheiten) sowie größtenteils mittels schriftlicher Fragebögen. Kinder und Jugendliche ab 11 Jahren erhielten einen eigenen Fragebogen. In KiGGS Welle 1 dagegen erfolgten alle Befragungen mittels standardisierter Telefoninterviews. Aus der Literatur ist bekannt, dass sich das Antwortverhalten Befragter zwischen Interview- und Fragebogen-Modus systematisch unterscheiden kann [57]. So werden in Interviews Fragen tendenziell eher in Richtung des sozial Erwünschten oder zustimmend beantwortet [58] und Zahlenangaben eher gerundet [59] als in schriftlichen Befragungen. Antworten auf Fragen nach sozial nicht bewerteten Merkmalen hingegen sind durch ModusEffekte kaum betroffen [60, 61]. Auch wenn die Auswirkungen auf die Ergebnisse nach ersten Analysen nicht als gravierend anzusehen sind, ist doch bei Prävalenzvergleichen zwischen der KiGGS-Basiserhebung und KiGGS Welle 1 stets zu diskutieren, ob die Trendergebnisse im jeweiligen inhaltlichen Kontext möglicherweise ganz oder teilweise durch den Befragungsmodus erklärt werden können.

\section{Schlussfolgerungen und Ausblick}

Aufgrund ihres Mischdesigns sowie der teilweise geringen und selektiven Teilnahmebereitschaft der Eingeladenen unterliegt die Stichprobe von KiGGS Welle 1 verschiedenen Limitationen, die so weit wie möglich durch Gewichtungsfaktoren ausgeglichen werden. Das nicht unübliche Mischdesign bietet auf der anderen Seite Effizienzgewinne durch die gleichzeitige Ermöglichung von Querschnitt-, Trend- und Lebensverlaufsanalysen. Die Methoden erfordern bei allen inhaltli- chen Datenauswertungen eine sorgfältige Diskussion und Interpretation der Ergebnisse.

Die in Vorbereitung befindliche Studie KiGGS Welle 2 wird - ähnlich der KiGGS-Basiserhebung - Befragungsund Untersuchungselemente aufweisen. Die Befragungen erfolgen ähnlich wie in der KiGGS-Basiserhebung mittels schriftlicher Selbstausfüllfragebögen. Im Altersbereich 0 bis 17 Jahre wird - wie bereits 2007 in der Gesamtplanung für das Studiendesign der KiGGS-Studie festgelegt - eine neue bevölkerungsbezogene Querschnittstichprobe zufällig aus Melderegistern ausgewählt und eingeladen. Alle Prävalenzschätzungen sollen aus dieser Querschnittstichprobe abgeleitet werden. Darüber hinaus werden alle Teilnehmenden der KiGGS-Basiserhebung, die dann 10 bis 27 Jahre alt sein werden, erneut zur Teilnahme eingeladen. Die Kohortenstichprobe soll ausschließlich zur Analyse von Entwicklungsverläufen und möglicherweise kausalen Zusammenhängen ausgewertet werden. Durch telefonischen oder persönlichen Kontakt mit Eingeladenen, die zunächst nicht antworten, wird eine möglichst hohe Teilnahmequote in beiden Stichproben sichergestellt werden.

\section{Korrespondenzadresse}

\section{Lange}

Abteilung für Epidemiologie und

Gesundheitsberichterstattung

Robert Koch-Institut

General-Pape-Straße 62-66, 12101 Berlin

LangeM@rki.de

\section{Einhaltung ethischer Richtlinien}

Interessenkonflikt. M. Lange, H.G. Butschalowsky, F. Jentsch, R. Kuhnert, A. Schaffrath Rosario, M. Schlaud und P. Kamtsiuris geben an, dass kein Interessenkonflikt besteht.

Finanzierung der Studie. Die Studie wurde mit Mitteln des Robert Koch-Institutes und des Bundesministeriums für Gesundheit finanziert

\section{Literatur}

1. Kurth B-M (2012) Das Gesundheitsmonitoring was es enthält und wie es genutzt werden kann. Public Health Forum 20:4.e1-4.e3 
2. Kurth B-M, Lange C, Kamtsiuris P, Hölling H (2009) Gesundheitsmonitoring am Robert Koch-Institut. Sachstand und Perspektiven. Bundesgesundheitsbl Gesundheitsforsch Gesundheitsschutz 52:557-570

3. Hölling $H$, Schlack R, Kamtsiuris $P$, Butschalowsky H, Schlaud M, Kurth BM (2012) Die KiGGS-Studie: Bundesweit repräsentative Längs- und Querschnittstudie zur Gesundheit von Kindern und Jugendlichen im Rahmen des Gesundheitsmonitorings am Robert Koch-Institut. Bundesgesundheitsbl Gesundheitsforsch Gesundheitsschutz 55:836-842

4. Hölling $H$, Kamtsiuris $P$, Lange $M$, Thierfelder $W$, Thamm M, Schlack R (2007) Der Kinder- und Jugendgesundheitssurvey (KiGGS): Studienmanagement und Durchführung der Feldarbeit. Bundesgesundheitsbl Gesundheitsforsch Gesundheitsschutz 50:557-566

5. Kamtsiuris P, Lange M, Schaffrath Rosario A (2007) Der Kinder- und Jugendgesundheitssurvey (KiGGS): Stichprobendesign, Response und Nonresponse-Analyse. Bundesgesundheitsbl Gesundheitsforsch Gesundheitsschutz 50:547-556

6. Kurth B-M, Kamtsiuris P, Hölling $\mathrm{H}$ et al (2008) The challenge of comprehensively mapping children's health in a nationwide health survey: design of the German KiGGS-Study. BMC Public Health 8:196

7. Aschpurwis + Behren GmbH (Hrsg) (2001) BIK Regionen. Ballungsräume, Stadtregionen, Mittel-/ Unterzentrengebiete. Methodenbeschreibung zur Aktualisierung 2000. Hamburg

8. Robert Koch-Institut (Hrsg) (2011) KiGGS - Kinderund Jugendgesundheitsstudie Welle 1. Projektbeschreibung. Robert Koch-Institut, Berlin

9. Robert Koch-Institut (Hrsg) (2011) Studie zur Gesundheit von Kindern und Jugendlichen in Deutschland (KiGGS Welle 1): Durchführung der Telefoninterviews. Epidemiol Bull 14:109

10. Robert Koch-Institut (Hrsg) (2006) Erste Ergebnisse der KiGGS-Studie zur Gesundheit von Kindern und Jugendlichen in Deutschland. Robert Koch-Institut, Berlin

11. Robert Koch-Institut (Hrsg) (2012) Studie zur Gesundheit von Kindern und Jugendlichen in Deutschland (KiGGS Welle 1): Durchführung einer Probanden-Zufriedenheitsbefragung. Epidemiol Bull 14:121

12. Biemer PP, Lyberg LE (2003) Introduction to survey quality. Wiley, New York

13. Brauns H, Scherer S, Steinmann S (2003) The CASMIN educational classification in international comparative research. In: Hoffmeyer-Zlotnik J, Wolf $\mathrm{CH}$ (Hrsg) Advances in cross-national comparison. A european working book for demographic and socio-economic variables. Kluwer Academic/ Plenum Publishers, Amsterdam, S 221-244

14. Hölling H, Erhart M, Ravens-Sieberer U, Schlack R (2007) Verhaltensauffälligkeiten bei Kindern und Jugendlichen. Erste Ergebnisse aus dem Kinderund Jugendgesundheitssurvey (KiGGS). Bundesgesundheitsbl Gesundheitsforsch Gesundheitsschutz 50:784-793

15. Statistisches Bundesamt (2010) Leben in Deutschland - Ergebnisse des Mikrozensus. Eigenverlag, Wiesbaden

16. Bundesministerium für Gesundheit (2011) Gesetzliche Krankenversicherung. Mitglieder, mitversicherte Angehörige und Krankenstand. Jahresdurchschnitt 2010 (Ergebnisse der GKV-Statistik KM1/13). Stand. 1. April 2011. Eigenverlag, Bonn/ Berlin
17. Verband der Privaten Krankenversicherung (2011) Zahlenbericht der Privaten Krankenversicherung 2010/2011. Köln

18. Wagner G, Frick J, Schupp J (2007) The German Socio-Econimic Panel Study (SOEP) - scope, evolution and enhancements. Schmollers Jahrbuch 127:139-169

19. Sikorski U, Horneffer B, Kuchler B (2009) Die Längsschnittdaten von EU-SILC - Datenstruktur und Hochrechnungsverfahren. Wirtschaft Statistik (11):1117-1126

20. Tolonen $\mathrm{H}$, Koponen P, Aromaa A et al (2008) Review of health examination surveys in Europe. Publications of the National Public Health Institute 18/2008, Helsinki

21. Edwards B (2012) Growing up in Australia: the longitudinal study of Australian children. The first decade of life. Family Matters 91:7-17

22. Morton SM, Atatoa Carr PE, Grant CC et al (2012) Cohort profile: growing up in New Zealand. Int J Epidemiol 42:1-11

23. Harris K (2011) Design features of add health. Carolina Population Center, University of North Carolina at Chapel Hill. http://www.cpc.unc.edu/ projects/addhealth/data/guides/design\%20paper\%20WI-IV.pdf. Zugegriffen: 24. Feb. 2014

24. Harris KM, Tucker Halpern C, Hussy J et al (2013) Social, behavioral, and genetic linkages from adolescence into adulthood. Am J Public Health 103:S25-S32

25. Harris KM, Gordon-Larsoen P, Chantala K, Udry JR (2006) Longitudinal trends in race/ethnic disparities in leading health indicators from adolescence to young adulthood. Arch Pediatr Adolesc Med 160:74-81

26. Lee H, Lee D, Guo G, Harris KM (2011) Trends in body mass index in adolescence and young adulthood in the United States: 1959-2002. J Adolesc Health 49:601-608

27. Morris M, Handcock MS, Miller WC et al (2006) Prevalence of HIV infection among young adults in the United States: results from the add health study. Am J Public Health 96:1091-1097

28. Resnick MD, Bearman PS, Blum RW et al (2010) Protecting adolescents from harm. Findings from the national longitudinal study on adolescent health. JAMA 278:823-829

29. Von der Lippe P, Kladropa A (2002) Repräsentativität von Stichproben. Marketing 24:227-238

30. Aschpurwis + Behren GmbH (Hrsg) (2010) BIK-Regionen. Ballungsräume, Stadtregionen, Mittel-/ Unterzentrengebiete. Methodenbeschreibung zur Aktualisierung 2010. Hamburg

31. Kamtsiuris $P$, Lange M, Hoffmann R et al (2013) Die erste Welle der Studie zur Gesundheit Erwachsener in Deutschland (DEGS1): Stichprobendesign, Response, Gewichtung und Repräsentativität. Bundesgesundheitsbl Gesundheitsforsch Gesundheitsschutz 56:620-630

32. Robert Koch-Institut (Hrsg) (2011) Daten und Fakten: Ergebnisse der Studie "Gesundheit in Deutschland aktuell 2009". Beiträge zur Gesundheitsberichterstattung des Bundes. Robert Koch-Institut, Berlin

33. Häder M (2006) Empirische Sozialforschung. Eine Einführung. VS Verlag für Sozialwissenschaften, Wiesbaden

34. Engel U, Bartsch S, Schnabel C, Vehre H (2012) Wissenschaftliche Umfragen. Methoden und Fehlerquellen. Campus, Frankfurt a. M.

35. Esser H (1986) Über die Teilnahme an Befragungen. ZUMA Nachrichten 18:38-47
36. Groves R, Cialdini R, Couper M (1992) Understanding the decision to participate in a survey. Public Opin Quarterly 56:475-495

37. Dillman DA (2000) Mail and internet surveys. The tailored design method. Wiley, New York

38. Ajzen I (1991) The theory of planned behavior. Organ Behav Hum Decis Processes 50:179-211

39. Groves R, Couper M (1998) Nonresponse in household interview surveys. Wiley, New York

40. Schnell R, Esser E, Hill PB (2008) Methoden der empirischen Sozialforschung. Oldenbourg, München

41. Stang A (2003) Nonresponse-Research - an underdeveloped field in epidemiology. Eur J Epidemiol 18:929-931

42. Curtin L, Mohadjer L, Dohrmann S et al (2012) The national health and nutrition examination survey: sample design, 1999-2006. National center for health statistics. Vital Health Statistics 2:1-39

43. Blumenberg M, Gummer T (2013) Gewichtung in der German Longitudinal Election Study 2009. GESIS-Technical Reports 2013/19. GESIS - Leibniz-Institut für Sozialwissenschaften, Köln

44. Kroh M, Spieß M (2008) Documentation of sample sizes and panel attrition in the German Socio Economic Panel (SOEP) (1984 until 2007). Data Documentation DIW Berlin Nr. 39. Deutsches Institut für Wirtschaftsforschung, Berlin

45. Schnell R (1993) Die Homogenität sozialer Kategorien als Voraussetzung für "Repräsentativität" und Gewichtungsverfahren. Z Soziol 22:16-32

46. Gabler S (2004) Gewichtungsprobleme in der Datenanalyse. Kölner Z Soziologie Sozialpsychologie Sonderheft 44:128-147

47. Lipps $O$ (2007) Attrition in the Swiss Household Panel. Methoden - Daten - Analysen 1:45-68

48. Vandecasteele L, Debels A (2006) Attrition in panel data: the effectiveness of weighting. Eur Sociol Rev 23:81-97

49. Särndal C-E, Lundström S (2005) Estimation in surveys with nonresponse. Wiley, New York

50. Robins JM, Rotnitzky A, Zhao L-P (1995) Analysis of semiparametric regression models for repeated outcomes in the presence of missing data. J Am Statistical Assoc 90:106-121

51. Philipson P, Ho W, Henderson R (2008) Comparative review of methods for handling drop-out in longitudinal studies. Stat Med 27:6276-6298

52. Fitzmaurice G, Davidian M, Verbeke G, Molenberghs GH (2009) Longitudinal data analysis. Handbooks of modern statistical methods. Chapman Hall/CRC, Boca Raton

53. Little J, Rubin D (1987) Statistical analysis with missing data. Wiley, New York

54. Arzheimer K (2009) Mehr Nutzen als Schaden? Wirkung von Gewichtungsverfahren. In: Schoen $\mathrm{H}$, Rattinger $\mathrm{H}$, Oscar W (Hrsg) Vom Interview zur Analyse. Methodische Aspekte der Einstellungsund Wahlforschung. Nomos, Baden-Baden, S 361 388

55. Golinelli D, Ridgeway G, Rhoades H, Tucker J, Wenzel S (2012) Bias and variance trade-offs when combining propensity score weighting and regression: with an application to HIV status and homeless men. Health Serv Outcomes Res Method 12:104-118

56. Hainmueller J (2012) Entropy balancing for causal effects: a multivariate reweighting method to produce balanced samples in observation studies. Political Analysis 20:25-46

57. De Leeuw E (2005) To mix or not to mix data collection modes in surveys. J Off Statistics 21:233255 
58. Moss S (2008) Acquiescence bias. Unter: http:// www.psych-it.com.au/Psychlopedia/article. asp?id=154. Zugegriffen: 24. Feb. 2014

59. Kraus F, Steiner V (1995) Modelling heaping effects in unemployment duration models - with an application to retrospective event data in the German Socio-Economic panel. Discussion Paper No. 95-09. Zentrum für Europäische Wirtschaftsforschung, Mannheim

60. Vissing N, Jensen S, Bisgaard H (2012) Validity of information on atopic disease and other illness in young children reported by parents in a prospective birth cohort study. BMC Med Res Method 12:1471-2288

61. Christensen A, Ekholm O, Glümer C, Juel K (2014) Effect of survey mode on response patterns: comparison of face-to-face and self-administered modes in health surveys. Eur J Public Health. 24:327332 